\title{
Quand la causative et la réflexive se rencontrent... Les différentes valeurs de la construction en se faire
}

\author{
Christel Le Bellec \\ Praxiling UMR 5267 CNRS - Université Paul-Valéry Montpellier 3 \\ christel.le-bellec@univ-monpt3.fr
}

\section{Introduction}

La construction en se faire + infinitif ne s'analyse pas systématiquement comme l'association d'une structure causative où le verbe faire permettrait d'augmenter la valence du verbe et d'une structure réflexive dans laquelle la particule réflexive aurait pour effet de réduire au contraire la valence verbale. En réalité, la situation est beaucoup plus complexe, car, si effectivement, sur le plan sémanticosyntaxique, la construction en se faire se laisse décrire en termes de causation réflexive dans de nombreux cas, elle peut parfois être décrite en termes de passivation.

Dans un premier temps, nous étudierons l'évolution de se faire, nous verrons ainsi comment une construction causative originelle a évolué vers une construction d'interprétation passive, puis, dans un second temps, nous aborderons les différentes valeurs de se faire et en étudierons les particularités syntaxiques et sémantiques : nous partirons ainsi de la lecture causative pour aller vers les différentes lectures passives.

\section{De la construction causative à la construction passive}

\subsection{De la grammaticalisation de faire causatif...}

Nous rappelons ici brièvement les caractéristiques de la grammaticalisation de faire causatif, en termes de désémantisation et de décatégorisation de l'auxiliaire faire (section 1.1.1), puis en termes de soudure de faire et du verbe à l'infinitif (section 1.1.2).

\subsubsection{Désémantisation et décatégorisation}

La grammaticalisation est définie par Kurylowicz (1965) comme « un processus qui consiste à convertir graduellement des entrées lexicales pleines en éléments grammaticaux, voire en morphèmes ». La grammaticalisation implique notamment deux processus que l'on peut vérifier pour la construction causative : la désémantisation et la décatégorisation (Bezinska, 2008).

La désémantisation est définie comme le fait que : «le verbe se vide de son sens plein pour acquérir un sens fonctionnel, grammatical » (Lamiroy, 1999). En effet, le verbe faire dans la construction causative se décharge de son contenu lexical ( $c f$. (1a)) pour se réduire à l'expression de la causation (signifiant 'faire en sorte que quelque chose ait lieu' ou 'être la cause de') $(c f .(1 \mathrm{~b}))^{1}$ :

(1) a. Je fais mon devoir (= je rédige mon devoir)

b. Je fais corriger mon devoir (par Jean) (= je fais en sorte que Jean corrige mon devoir)

De plus, la grammaticalisation implique une « décatégorisation » que Lehmann (1995) décrit comme une « dégénérescence morphologique », dans la mesure où des unités de $1^{\text {er }}$ niveau (noms, verbes, adjectifs, etc.) Se transforment en unités de $2^{\text {nd }}$ niveau (adverbes, auxiliaires, affixes verbaux, etc.).

Une des conséquences de cette décatégorisation est que le verbe perd en capacité de sélection (Lamiroy, 1999) et qu'il tend à s'associer à des verbes non finis, tels que l'infinitif. C'est ce que l'on peut observer pour la construction en faire + infinitif, puisque c'est l'ensemble du groupe qui récupère les propriétés de 
sélection, en raison de la perte d'autonomie du verbe faire. Cette décatégorisation est fortement liée à la soudure des deux verbes comme nous allons le vérifier ci-après ${ }^{2}$.

\subsubsection{Soudure des deux verbes}

La perte d'autonomie de faire a pour conséquence la coalescence de ce dernier avec l'infinitif qui le suit, car comme le signale Lamiroy (1999), « moins un élément a de substance propre, plus il aura tendance à s'appuyer sur son entourage ».

Ainsi, les clitiques s'antéposent à l'ensemble du groupe verbal et non au seul verbe à l'infinitif :

(2) a. Je $l$ 'ai fait dormir

b. *J'ai fait le dormir

Néanmoins, le fait que l'on puisse insérer certains éléments entre le verbe faire et l'infinitif, tels que le pronom sujet inversé dans les phrases interrogatives (3a), l'élément négatif pas (4a), l'élément tout (5a) et certains modifieurs adverbiaux (6a), n'est pas un indicateur d'un manque de cohésion mais au contraire, cela témoigne de la correspondance entre la causative et les verbes formés de l'auxiliaire être ou avoir suivis d'un participe passé, comme en $(3 b, 4 b, 5 b, 6 b)$ :

(3) a. Fera-t-il partir Marie ?

b. Marie est-elle partie?

(4) a. On ne fera pas partir Jean

b. Jean n'est pas parti

(5) a. Il fera tout sauter

b. Marie a tout entendu

(6) a. Ils le feront sans doute pleurer

b. Jean a sans doute pleuré

Un autre indice de soudure syntaxique réside dans le fait que la causative en faire constitue une seule unité phrastique par opposition aux phrases à complétives qui contiennent deux prédicats bien distincts. Cela a pour conséquence que la construction en faire + infinitif a un comportement limité sur divers plans syntaxiques et sémantiques, en raison de ses propriétés de mono-clausalité.

En effet, le complément de faire ne peut pas être repris par un pronom car ce n'est pas un constituant, tandis que les complétives le peuvent (Vet, 1991 et Dik, $1980: 65$ ) :

(7) a. *Pierre a fait partir Jean et Marie l'a fait aussi

b. Jean a fait en sorte que Pierre chante et Marie l'a fait aussi

Le verbe à l'infinitif ne peut avoir de négation indépendamment de faire, tandis que les complétives peuvent être niées ; la structure en faire suivi d'un infinitif se comporte donc comme un verbe simple par rapport à la négation :

(8) a. *Jean fera ne pas travailler Pierre

b. Jean ne fera pas travailler Pierre

(9) a. Jean ne fera pas en sorte que Pierre travaille

b. Jean fera en sorte que Pierre ne travaille pas

Enfin, il est impossible d'associer faire, indépendamment du verbe à l'infinitif, à un autre verbe de sens aspectuel, alors que cela est possible avec les complétives :

(10) a. Jean continue de faire travailler Pierre b. *Jean fait continuer de travailler Pierre

(11) a. Jean continue de faire en sorte que Pierre travaille b. Jean fait en sorte que Pierre continue de travailler

Ainsi, le comportement de faire + infinitif reflète le haut degré de fusion des deux verbes de la construction causative. 


\section{$2.2 \quad$... à la grammaticalisation de se faire passif}

\subsubsection{Réanalyse de se faire}

Dans la construction causative, fruit d'une grammaticalisation du semi-auxiliaire faire, la particule réflexive se, indépendante de la construction, agit alors comme un vrai pronom réfléchi pour former des constructions à valeur causative réfléchie.

Concernant la valeur passive de se faire, la grammaticalisation va encore plus loin, puisque la particule réflexive est impliquée dans le changement, elle fait partie intégrante du semi-auxiliaire et l'ensemble donne ainsi lieu à une lecture passive.

Ainsi, d'après Kurylowicz (1965), la grammaticalisation implique le passage d'une catégorie lexicale à une catégorie grammaticale ( $c f$. faire causatif) ou d'une catégorie déjà grammaticale à une unité plus grammaticale encore.

En effet, si l'on part de la construction causative réfléchie, comme dans (12), on passe de deux éléments grammaticaux distincts portés par la particule réflexive et le verbe causatif (la réflexivité et la causation) à un élément grammatical unique (la passivation), représenté par l'ensemble se faire, comme dans (13) :

(12) Elle se fait vomir après chaque repas

(13) Il s'est fait poignarder en plein jour

Ainsi, le semi-auxiliaire se faire passif serait le résultat d'une réanalyse, c'est-à-dire d'une modification de sa structure sous-jacente sans changement de sa structure de surface, ce qui implique un reparenthésage des éléments (selon la définition de Langacker, 1977 : 58).

Cette réanalyse implique deux changements majeurs: d'une part, au niveau syntaxique, il y a resegmentation des éléments constitutifs du semi-auxiliaire, qui consiste en la fusion des deux opérateurs (le verbe faire et la particule réflexive) :

(14) [se] [faire] $>$ [se faire]

D'autre part, au niveau sémantique, il y a réinterprétation du semi-auxiliaire qui passe de la causation réflexive à la passivation :

(15) se réflexif + faire causatif $>$ se faire passif

En effet, lorsqu'on analyse une phrase comme celle en (13) (cf. Il s'est fait poignarder), ni la particule réflexive, ni le verbe faire ne s'analysent comme dans la construction causative : la particule ne joue plus son rôle réflexif (16a) et le verbe faire n'a plus de rôle causatif (16b) :

(16) a. *Il a fait poignarder lui-même

b. ?Il a fait en sorte qu'on le poignarde

Notons que cette réanalyse génère une ambiguïté entre la forme causative toujours vivante et la forme passive, dans la mesure où les deux interprétations cohabitent dans la langue, comme dans :

(17) a. Elle s'est fait remarquer par tout le monde malgré sa grande discrétion

b. Elle s'est fait remarquer exprès pour faire honte à ses parents

Par ailleurs, se faire passif est une construction moderne, comme nous avons pu le constater à travers l'examen d'un grand nombre d'occurrences de se faire à sens passif dans Frantext. Il apparaît, en effet, qu'à partir du milieu du $20^{\mathrm{e}}$ siècle, le nombre d'occurrences pour un certain nombre de verbes (parmi les plus courants, comme se faire voler/piquer/écraser/gronder, etc.) se voit doubler et que, parallèlement à cela, cette même période voit apparaitre en tant que verbe à l'infinitif dans se faire passif un grand nombre de nouveaux emplois au sens figuré (comme nous le verrons dans la section 2.4.2.). 


\title{
2.2.2 Les raisons du changement
}

Les principales raisons qui sont à l'origine d'un tel changement sont d'ordre sémantico-syntaxique et expressif.

En effet, la construction causative, comme : Elle s'est fait ramener par un taxi, en plus du sens causatif qu'elle manifeste (= elle a fait en sorte qu'un taxi la ramène), présente également un sens passif intrinsèque à la construction car la particule réflexive transmet le rôle de Patient au sujet de la construction (= elle a été ramenée par un taxi) en plus de son rôle de Causateur. Par la suite, le sens de la construction aurait dérivé vers des situations sans Causateur, comme Elle s'est fait attaquer par un chien à côté de Elle a été attaquée par un chien, où le sujet n'a plus que le rôle de Patient, entrant donc en concurrence avec le passif canonique (Le Bellec, 2013).

À l'instar des expressions verbales figées, la construction en se faire, prise dans son ensemble, s'est écartée de la signification originelle de ses composantes pour prendre un sens purement passif, comme l'indiquent Lamiroy et al. (2010) au sujet des expressions verbales figées :

\begin{abstract}
«L'utilisation répétée de certaines séquences mènerait à leur automatisation, entraînant un traitement de la séquence en tant qu'unité soudée : autrement dit, à force d'aligner systématiquement les mêmes éléments consécutifs dans la chaîne linéaire, le sens individuel dans des mots au départ autonomes finirait pas ne plus être perçu au profit d'un sens global. » (p. 4)
\end{abstract}

Par ailleurs, l'affinité de la particule réflexive avec certaines valeurs aspectuelles, la valeur d'aspect inaccompli inhérente à l'infinitif et la malléabilité syntaxique de la construction en font une structure idéale pour pallier certains déficits aspectuels et syntaxiques du passif canonique, comme nous le verrons plus loin.

En outre, l'utilisation de la construction en se faire, pour des emplois passifs, s'explique par le besoin d'expressivité (Meillet, 1912 ; Frei, 1929) ou encore le besoin d' " extravagance » (Haspelmath, 1999), qui consiste pour le locuteur à se faire remarquer, à attirer l'attention de l'interlocuteur sur sa façon de parler, donc à dire les choses de façon plus originale, plus frappante. Ce désir d'expressivité du locuteur est lié au phénomène de subjectivation (Traugott, 1980) qui est un processus sémantique et pragmatique par lequel le locuteur marque son implication forte et guide en quelque sorte l'interlocuteur vers une interprétation dérivée de l'énoncé.

La construction passive canonique s'est certainement avérée trop neutre pour exprimer certaines situations à sens passif, ce qui l'a conduite à être remplacée par le passif en se faire, qui est plus « expressif » et « extravagant », dans la mesure où il permet au locuteur de manifester son implication dans l'énoncé et son empathie envers le référent du sujet, tandis que être + participe passé, comme l'affirme Raineri (2010) signale le désinvestissement du locuteur au profit des faits bruts. C'est la raison pour laquelle on trouve un grand nombre de passifs en se faire notamment dans les titres, dont l'objectif est d'attirer l'attention du lecteur (à travers les occurrences relevées grâce au moteur de recherche Google).

Voici quelques exemples de titres pour lesquels des équivalents au passif canonique seraient moins « expressifs» et feraient preuve de plus de détachement, de neutralité au regard des faits décrits ou apparaîtraient moins «sensationnels » (cf. ex. 22), comme on peut le constater à travers les paires de phrases suivantes :

(18) a. Mon chat de 1 an $1 / 2$ s'est fait écrasé vendredi. j'arrive pas à remonter la pente. besoin d'aide (titre d'une discussion tiré du forum aufeminin.com)

b. Mon chat de 1 an $1 / 2$ a été écrasé vendredi. (...)

(19) a. Une femme se fait renverser par un taxi au Brésil (titre d'une vidéo sur Spion.com) ${ }^{3}$

b. Une femme est renversée par un taxi

(20) a. En direct il se fait mordre par un requin ! (dailymotion.com)

b. En direct il est mordu par un requin ! 
Comme l'affirme Raineri (2010), l'utilisation de la construction en se faire est alors privilégiée pour dire les choses avec empathie ou pour attirer la compassion de l'interlocuteur. Par exemple :

(21) a. Je me suis fait virer de mon bureau

b. J'ai été viré de mon bureau

Dans la première phrase, le locuteur parle avec plus d'implication, il se sent lésé par ce qui lui est arrivé, tandis que la seconde est plus neutre et distante, le locuteur ne s'implique pas dans son énoncé, il ne prend pas part à la situation et la regarde avec plus de recul et de détachement.

Enfin, les deux principes (sémantico-syntaxique et expressif) sont liés : le déplacement sémantique est le résultat d'un usage social du langage, c'est un moyen pour forger un énoncé qui attire l'attention (Marchello-Nizia, 2006).

En conséquence de ce changement (la réanalyse de se faire), le français se retrouve avec une forme passive supplémentaire qui va suppléer, d'une part, les déficiences du passif canonique pour certains emplois (voir section 2.3.) et qui, d'autre part, va se trouver en concurrence avec cette dernière (voir section 2.4.).

\section{Les valeurs de se faire}

Nous avons vu que se faire suivi de l'infinitif avait une double existence et que sa valeur passive était dérivée de sa valeur causative primaire.

À présent, nous allons examiner ses différentes valeurs. Nous commencerons par une discussion sur la difficulté du découpage des valeurs de se faire, puis nous partirons de la valeur causative réfléchie, pour aller vers la valeur causative-passive, en passant par la valeur purement passive et enfin terminerons avec la valeur spontanée de se faire.

\subsection{Le découpage des valeurs de se faire}

Lorsque l'on souhaite procéder au découpage des différentes valeurs de se faire, on se heurte à la difficulté liée au chevauchement des niveaux syntaxique et sémantique. Au niveau sémantique, on distingue, en effet, deux grandes valeurs, puis, sur le plan syntaxique, on distingue trois types de structures selon le nombre d'arguments autour du verbe. Les deux se recoupent mais également se séparent à différents endroits.

Ainsi, au niveau syntaxique, nous distinguons trois types :

- se faire + verbe intransitif (par exemple: Elle se fait bronzer au bord de la piscine) : cette structure ne peut donner lieu qu'à la lecture causative, la valeur passive est impossible car elle nécessite un second actant ;

- se faire + verbe transitif (par exemple : Il se fait habiller par un grand couturier d'interprétation causative et Elle s'est fait agresser par un chien errant d'interprétation passive) : les valeurs passive ou causative sont mises en évidence grâce au sémantisme du verbe à l'infinitif et au contexte en jeu ;

- se faire + verbe doublement transitif (par exemple : Elle s'est fait refaire les seins pour la valeur causative et Je me suis fait voler mon sac à main, pour la valeur passive) là aussi le contexte permet de mettre en évidence l'une ou l'autre valeur; de plus, ce type de structure met fréquemment en jeu des noms de parties du corps tant avec la valeur causative que passive.

En revanche, au niveau sémantique, nous distinguons deux valeurs : la valeur originelle causative et la valeur dérivée passive.

Comme nous venons de le voir, les deux valeurs coexistent pour des verbes transitifs et doublement transitifs, tandis qu'elle reste causative pour les verbes intransitifs. 
Si la construction passive à verbe transitif est concurrente au passif canonique, en revanche, la structure doublement transitive à valeur passive acquiert un statut différent dans la mesure où le passif canonique n'existe pas pour ce type de verbes, elle devient donc supplétive par rapport à ce dernier.

Par ailleurs, parmi les constructions à valeur passive s'ajoute le passif «spontané » à sujet inanimé, qui est à rapprocher de la construction pronominale neutre (du type : La porte s'est ouverte).

Nous prenons donc le parti de distinguer quatre constructions en se faire : le causatif réfléchi, le causatifpassif (pour les verbes doublement transitifs qui inclut les deux valeurs : causative et passive), le passif et le spontané.

\subsection{Se faire causatif réfléchi}

Lorsque se faire fonctionne comme un causatif réfléchi, il constitue alors l'association des diathèses causative et réfléchie.

La particule réflexive «se » agit comme un véritable pronom à valeur réfléchie car le procès se retourne sur le sujet. Cette propriété est identifiable grâce à la possibilité d'ajouter la forme renforcée « lui-même » (22b) et à la possibilité de le remplacer par un pronom clitique d'une autre personne (22c) et (23b) (ce qui est impossible pour la construction en se faire à valeur passive) :

(22) a. Ils se faisaient inviter partout, les voyages au bout du monde, les week-ends familiaux, les dîners secrets. (Orsenna, E. Grand amour, 1993, p. 243)

b. Ils se faisaient inviter eux-mêmes partout (...)

c. Ils nous faisaient inviter partout (...)

(23) a. L'Empereur se faisait habiller par ses valets (Rambaud, P. La bataille, 1997, p.276)

b. L'Empereur le faisait habiller par ses valets

En outre, le sens causatif est nettement perceptible, car le référent du sujet représente l'instigateur du procès, il fait en sorte que la situation ait lieu, l'adjonction de s'efforcer de permet de mettre en évidence ce rôle d'instigateur (François, 2001) :

(24) Ils s'efforçaient de se faire inviter partout

On trouve, dans ce type de construction, un petit nombre de verbes intransitifs, parmi ceux-ci principalement des verbes de changement d'état, du type : rire, bronzer, maigrir, vomir, exploser, avorter, saigner, etc. (Novakova, 2008). Il s'agit le plus souvent de verbes processifs, donc non-agentifs qui décrivent une situation provenant du référent du sujet qui survient sans contrôle. Le verbe faire apporte alors à ces verbes processifs le trait de contrôle qui leur manque.

(25) Lorsque la Reine laisse aller une bouchée de trop, elle monte sans façon se faire vomir dans les toilettes. (Pennac, D., La petite marchande de prose, 1989, p. 346)

(26) (...) ou se faire bronzer avec des lampes... (Djian, P. 37.2 le matin, 1985, p. 286)

De plus, les verbes intransitifs donnent forcément lieu à l'interprétation causative réfléchie puisque ce type de verbes ne peut entrer dans la construction passive.

En revanche, les constructions en se faire suivi d'un verbe transitif peuvent donner lieu à ambiguïté puisque la valeur passive exploite aussi abondamment ce type de verbes :

(27) Elles se font photographier

(28) Elle se fit embaucher comme professeur d'anglais (Roze, P. Le chasseur zéro, 1996, p. 15)

Néanmoins, différents indices nous permettent de vérifier l'interprétation causative réfléchie face à l'interprétation passive, portant simultanément sur le rôle sémantique du sujet, la fonction du réflexif et le sens du verbe faire (Le Bellec, 2013) :

- le sujet est agentif, il est l'instigateur de l'action représentée par le verbe à l'infinitif, il fait en sorte que l'événement ait lieu et à ce titre, il a un double rôle sémantique : instigateur et patient ; 
- la particule réflexive joue pleinement son rôle réflexif : le procès du verbe à l'infinitif se retourne sur le sujet, c'est pourquoi ce dernier acquiert le rôle de patient en plus de celui d'instigateur ;

- le verbe faire a un sens causatif, dans la mesure où le référent du sujet fait en sorte que l'événement ait lieu, c'est ce qui lui permet d'acquérir le rôle d'instigateur.

De plus, la valeur causative est souvent perceptible grâce à un certain nombre d'indices, comme le contexte, les connaissances du monde et le sémantisme du verbe. Ces éléments permettent de lever l'éventuelle équivoque (nous soulignons les indices contextuels permettant d'orienter vers une interprétation causative) :

(29) Sa fille qui se prénommait sur l'état civil « Thérèse », mais se faisait appeler « Micheline ». (Boudard, A., Mourir d'enfance, 1995, p. 130)

(30) Nicolas, assis à l'arrière, trouvait difficile de se faire entendre à cause du bruit de la soufflerie. (Carrere, E. La classe de neige, 1995, p. 8 )

(31) Jean est mort, je le sais, j'en suis sûre. Il l'a fait exprès, il disait la mort c'est gai, une cabriole. Il s'est fait tuer. Exprès. Contre moi.

(32) La cliente qui va se faire avorter croise dans l'ascenseur celle qui va se faire inséminer. (Sollers. P, Le secret, 1993, p. 63)

(33) Ce même automne 1939, l'admirable Freud, exilé à Londres, n'en pouvant plus de souffrance, se fait injecter, avec la permission de sa fille, une dose mortelle de morphine par son médecin (Sollers, P. Le secret, 1993, p. 196)

(34) Mais il estimait sans doute qu'il valait mieux se faire oublier. (Rouaud, J., Les champs d'honneur, 1990, p. 56)

(35) Pour se marier religieusement, Clara a dû se faire baptiser. (Pennac, D. La petite marchande de prose, 1989, p. 58)

\subsection{Se faire causatif-passif}

Les verbes doublement transitifs construits avec se faire peuvent donner lieu à l'interprétation causative ou passive. Le type de verbe privilégié dans ce type de construction est représenté essentiellement par Blanche-Benveniste (2001) :

- des verbes de donation ou de privation (ex : se faire offrir/servir/voler qqch., etc.)

- des verbes de communication (ex : se faire communiquer qqch., etc.)

- des verbes à datif partitif (mention d'une partie du corps du sujet) (ex : se faire couper les cheveux, etc.).

Les constructions à datif partitif (où l'objet direct représente une partie du corps du sujet) sont parallèles aux constructions réflexives non causatives où se peut être considéré comme un marqueur de possession inaliénable en raison de l'impossibilité d'avoir un objet direct déterminé par un possessif en présence de se :

(36) a. Elle va se faire couper les cheveux

b. *Elle va se faire couper ses cheveux

(37) a. Elle se lave les cheveux

b. *Elle se lave ses cheveux

Le sujet de la construction représente le destinataire (bénéficiaire ou victime selon le sens du verbe et le contexte) ou le possesseur du référent de l'objet du verbe (et non le patient comme dans les constructions transitives), car il correspond à un objet indirect dans la structure active :

(38) a. Il se fit amener son cheval gris le plus docile (Rambaud, P., La bataille, 1997, p. 193)

b. On lui amena son cheval gris le plus docile

(39) a. Malade, enflée, près de mourir, Thérésa se fait accorder une promenade dans la cour de la prison. (Kristeva, J., Les samouraïs, 1990, p. 180)

b. On accorde à Thérésa une promenade dans la cour de la prison

Concernant la valeur passive de cette construction, elle a un statut particulier dans la mesure où elle pallie l'impossibilité syntaxique pour le passif canonique de topicaliser un argument qui reçoit normalement la 
fonction d'objet indirect, grâce à sa plus grande souplesse syntaxique (François, 2001). Elle est donc supplétive par rapport au passif en être, puisque cette dernière est impossible :

(40) a. Il venait de se faire mordre la jambe par un serpent à sonnettes (Djian, P. 37.2 le matin, 1985, p. 137) b. *il venait d'être mordu la jambe

(41) a. Les précautions à prendre pour éviter de se faire voler ses chaussures. (Simon, C., L'acacia, 1989, p. 180)

b. *pour éviter d'être volé ses chaussures

Il est parfois délicat de statuer sur l'interprétation (causative ou passive) de la construction en se faire. D'après Bat-Zeev Shyldkrot (1999), la «causativité » d'une phrase peut être déterminée en fonction de l'existence d'un certain nombre de traits distinctifs correspondant aux traits du sujet vis-à-vis du procès exprimé par le verbe à l'infinitif, comme les traits suivants :

- il est l'instigateur du procès

- il participe au procès

- il exerce un contrôle sur le déroulement du procès

- il est responsable du procès

- il influe volontairement sur le procès.

La présence de l'ensemble de ces traits donnerait à la phrase une lecture causative, leur absence, en revanche, rendrait la phrase passive. Toutefois, ces traits sont rarement tous présents ou tous absents, il y a donc une sorte de continuum entre la valeur causative et la valeur passive de se faire. La causation implique de la passivité et la passivité n'exclut pas nécessairement la causation (surtout en l'absence de volonté de la part du référent du sujet).

C'est l'existence de certains traits mais pas de tous, qui rend la démarcation entre phrase passive et causative parfois incertaine et équivoque.

Ainsi, dans les exemples suivants, une majorité des traits distinctifs précédemment cités sont présents, ce qui nous permet de les considérer comme des phrases causatives (nous soulignons les indices contextuels qui rendent manifeste l'interprétation causative de la construction) :

(42) Il n'était plus rare qu'au milieu des batailles un conscrit exaspéré se faufile à l'abri d'un bosquet pour se faire sauter la cervelle. (Rambaud, P. La bataille, 1997, p. 222)

(43) Marie-Sandra l'a convaincue de se faire faire quelques injections de collagène (Brisac, G., Week-end de chasse à la mère, 1996, p. 192)

(44) Il se fait faire toutes les semaines, (...), des piqûres dans la verge pour la redurcir. (Guibert, H. À l'ami qui ne m'a pas sauvé la vie, 1990, p. 217)

(45) On a même réussi à se faire servir quelques cocktails envoûtants. (Hanska, E. Les amants foudroyés, 1984, p. 155)

À l'inverse, la construction est d'interprétation passive, lorsqu'aucun des traits distinctifs (ou une minorité) n'est manifesté, comme dans les exemples suivants :

(46) La plus grosse, un peu penaude, se faisait remonter les bretelles, parce qu'elle n'avait rien vu. (Brisac, G. Week-end de chasse à la mère, 1996, p. 53)

(47) Il disait qu'il en avait marre de venir à l'école pour se faire saloper des pantalons qu'il avait assez de mal à acheter (Carrère, E. La classe de neige, 1995, p. 55)

(48) L'aile semblait profondément touchée ; ce qui, pour un pigeon, est encore plus grave que pour un homme de se faire couper le sexe. (Jardin, A., Bille en tête, 1986, p. 176)

\subsection{Se faire passif}

Comme nous venons de le voir, plusieurs indices nous permettent d'identifier la construction en se faire d'interprétation passive face à la construction causative, bien que, pour certains auteurs, se faire soit une construction intrinsèquement causative (voir notamment Gaatone, 1983 et Tasmowski, 1987), ce à quoi nous nous opposons pour les raisons que nous avons vues dans la section 2.2 ainsi que celles que nous exposons dans cette section. 
Contrairement à la causative, on ne peut remplacer le sujet par un pronom d'une autre personne qui serait responsable du procès (Kupferman, 1995) :

(49) a. Il s'est fait agresser par un passant

b. ?On l'a fait agresser par un passant

La passivité, dans la passive en se faire, se caractérise par différents traits :

- le sujet porte uniquement le rôle de patient (il n'est pas l'instigateur de l'action)

- la particule réflexive n'a plus aucun rôle sémantique, elle est transparente sémantiquement (elle n'a plus de rôle réflexif)

- le verbe faire est transparent sémantiquement (il n'a plus de sens causatif).

Cette construction est très répandue en français oral et populaire. Elle est concurrente du passif canonique et non plus supplétive car elle est le plus souvent interchangeable avec ce dernier. Elle présente tout de même un changement de sens et/ou d'aspect important, ce qui rend la construction en se faire la plupart du temps plus naturelle que celle au passif canonique qui est parfois difficilement acceptable :

(50) a. Il se fait constamment enguirlander

b. ?Il est constamment enguirlandé

Nous allons voir ci-après les raisons qui rendent l'utilisation de se faire plus naturelle que le passif en être. Plusieurs aspects, liés entre eux, caractérisent en effet se faire passif et contraignent l'utilisation de cette construction face au passif canonique : l'aspect de la construction (section 3.4.1.), la manifestation du sens figuré (3.4.2.) et l'affectation du référent du sujet (section 3.4.3.).

\subsubsection{L'aspect de la construction}

Le passif canonique a tendance à présenter l'état résultant (Carlier, 2002), tandis que la construction en se faire permet de décrire non seulement l'entrée dans le procès mais aussi son déroulement (Spang-Hansen, 1967, Novakova, 2008). En effet, le procès est présenté comme inaccompli ou en cours de déroulement comme dans l'exemple suivant :

(51) a. Un jour elle se fera écraser comme tous ces hérissons (Beck, B., Stella Corfou, 1988, p. 59)

b. ?Un jour elle sera écrasée comme tous ces hérissons

L'association du passif avec des verbes marquant un aspect particulier contraint l'apparition de se faire passif face au passif en être :

- les périphrases temporelles du futur (proche) (aller + infinitif) (ex. 52) ou du passé proche (venir de + infinitif) (ex. 53) ou encore de verbes du type risquer de (ex. 54) ;

- les périphrases aspectuelles exprimant les différentes phases du procès (commencer à, être en train de, finir de, etc.) (ex. 55);

- après les verbes de perception qui impliquent un procès duratif (voir, entendre, regarder, etc.) (ex. 56).

(52) a. Tu vas te faire gronder si tu continues b. ?Tu vas être grondé si tu continues

(53) a. On venait de se faire coincer sérieux par des Teutons très aguerris dans les tempêtes (Boudard, A., Mourir d'enfance, 1995, p. 160)

b. ?On venait d'être coincés sérieux par des Teutons

(54) a. Ne mets pas tes mains sur les portes. Tu risques de te faire pincer très fort. (inscription sur les portes des wagons du métro parisien)

b. ?Tu risques d'être pincé très fort.

(55) a. Qui pouvait imaginer, (...), que des gens sont en train de se faire tuer au-dessus de Londres ? (D’Ormesson, J. Le bonheur à San Miniato, 1987, p. 118)

b. ?Qui pouvait imaginer que des gens sont en train d'être tués au-dessus de Londres?

(56) a. Je voyais des enfants se faire caresser par leur mère

b. ?Je voyais des enfants être caressés par leur mère 
Les raisons de cette aspectualité inhérente au passif en se faire et corrollairement à celui en être sont liées aux éléments qui composent ces tournures : le participe passé exprime d'ordinaire un procès accompli, tandis que l'infinitif désigne généralement un procès inaccompli; de plus, le passif en se faire a gardé une partie de la valeur inaccomplie de la particule réflexive, que l'on retrouve dans la construction moyenne (par ex. Ce pantalon se lave à la main).

\subsubsection{La manifestation du sens figuré}

On trouve fréquemment la construction passive en se faire avec des verbes pris au sens figuré, comme : se faire avoir, se faire pincer, etc. Le passif en être privilégie une lecture au sens propre, tandis que le passif en se faire manifeste exclusivement la lecture au sens figuré ${ }^{4}$, comme en témoignent les exemples suivants :

(57) a. Je suis sûr qu'on va se faire coincer... ! (Djian. P., 37.2 le matin, 1985, p. 106) b. ?Je suis sûr qu'on va être coincés... !

(58) a. Ils rentrèrent chez eux et se firent cueillir aussitôt. L'un et l'autre en prirent pour six mois (Rolin, J. L'organisation, 1996, p. 24)

b. ?Ils rentrèrent chez eux et furent cueillis aussitôt.

(59) Il quitta la salle et se fit refouler du jardin des mousses. (Guibert, H. À l'ami qui ne m'a pas sauvé la vie, 1990, p. 131)

(60) On se fait avoir à tous les coups! (Poirot-Delpech, B., L'été 36, 1984, p. 32)

Par analogie avec des verbes fréquemment agencés avec se faire, on associe des séries de verbes synonymes pris au sens figuré, comme :

- se faire arnaquer/piéger > se faire avoir/rouler, etc.

- se faire gronder/disputer > se faire enguirlander/remonter les bretelles/tirer les oreilles/incendier, etc.

- se faire arrêter/attraper/prendre > se faire pincer/coincer/cueillir/serrer/choper, etc.

- se faire tuer $>$ se faire descendre, etc.

- se faire battre > se faire écraser/dégommer, etc.

- se faire renvoyer > se faire refouler/éjecter, etc.

Le sens figuré se manifeste dans une proportion non négligeable dans le registre populaire ou familier, voire vulgaire, comme on peut le constater à travers les exemples suivants ${ }^{5}$ :

(61) Nous sommes plutôt cocorico va-t-en-guerre, on la ramène, on sort dehors et on se fait dérouiller salement comme à la der des ders. (Boudard. A., Mourir d'enfance, 1995, p. 67)

(62) Mets ta ceinture, elle a dit, on va se faire gauler par ces pourris de gendarmes... (Pouy. J-B., La clef des mensonges, 1988, p. 77)

(63) Ils ont dû se faire engueuler (Queffelec. Y., Les noces barbares, 1985, p. 193)

(64) (...) prépare le premier venu à se faire couillonner. (Giraud. R., Carrefour Buci, 1987, p. 20)

Par ailleurs, tous ces emplois au sens figuré ont en commun de présenter un patient affecté par l'action, point que nous allons discuter dans la sous-section suivante.

\subsubsection{L'affectation du référent du sujet}

Comme nous venons de le voir, se faire permet de présenter un procès inaccompli et permet également au verbe à l'infinitif de dénoter un sens figuré. En complément de cela, et à la différence du passif en être, se faire a tendance à marquer le référent du sujet comme grandement affecté par le procès ou fortement impliqué dans ce dernier, alors que le passif canonique est neutre de ce point de vue-là (Le Bellec, 2013) :

(65) a. Le démon me souffle que les fidèles qui se font brûler ne ressusciteront pas (Biancotti, H., Sans la miséricorde du Christ, 1985, p. 345)

b. ? Le démon me souffle que les fidèles qui sont brûlés ne ressusciteront pas 
Cette construction est obligatoirement construite avec un sujet affecté par l'événement (que cet affect soit positif ou négatif), puisqu'en effet les énoncés dont le sujet ne peut être envisagé comme affecté ne sont pas acceptables :

(66) *Jean s'est fait apercevoir par de nombreux collègues.

Cette plus forte implication dans le procès n'est pas obligatoirement détrimentaire pour le référent du sujet, elle peut aussi lui être bénéfique :

(67) Au terme d'un long discours et d'un bref débat, il se faisait acclamer par le Reichstag convoqué à Berlin (D’Ormesson. J, Tous les hommes sont fous, 1986, p. 184)

(68) On n'a jamais entendu dire qu'un type puisse se faire toucher par la Grâce sur le parking d'un supermarché (Djian., P., 37.2 le matin, 1985, p. 175)

Ainsi, ce rôle de Patient fortement impliqué dans le procès peut s'expliquer par son association avec l'un des deux rôles: détrimentaire ou bénéficiaire, par analogie avec se faire construit avec un verbe doublement transitif. Ce rôle de bénéficiaire ou détrimentaire s'ajouterait à celui de Patient dans la construction à verbe transitif et contribuerait donc à son interprétation de Patient fortement affecté par le procès.

Cette affectation du référent du sujet explique également pourquoi dans ce type de construction on trouve rarement des sujets inanimés. Ils peuvent en effet difficilement être affectés :

(69) ?Ma maison s'est fait repeindre

Cette plus forte implication dans le procès fait que cette construction est souvent employée pour décrire des procès où est en jeu la vie du référent du sujet, sa santé, ses biens, son intégrité, sa dignité, etc. :

(70) Ce furent des temps durs, où des chrétiens se faisaient massacrer, et massacraient les autres. (De Romilly.J., Les æufs de pâques, 1993, p. 216)

(71) Les officiels d'état se font huer et siffler dès qu'ils apparaissent. (Sollers. P., Le secret, 1993, p. 212)

(72) Lauzun se fait gronder, humilier, piétiner. Sans réagir. (Kristeva. J., Les samouraïs, 1990, p. 205)

(73) Elle était la seule qui avait failli se faire violer pour de bon. (Seguin. F., L'arme à gauche, 1990, p. 67)

(74) Un type se fait étaler de tout son long au moment où il allait jeter une canette dans la salle. (Manoeuvre. P., L'enfant du rock, 1985, p. 36)

(75) La petite souris, avant de se faire engloutir tout entière par le crotale (Aventin. C., Le cœur en poche, 1988, p. 90)

Ainsi, le référent du sujet peut être affecté de différentes manières (nous reprenons les grandes classes proposées dans Raineri (2013), que nous augmentons) :

- $\quad$ ses biens sont affectés (ex : se faire voler /cambrioler, etc.)

- sa situation sociale est affectée :

- par rejet (ex : se faire virer/renvoyer /éjecter/sortir/refouler /jeter/larguer, etc.)

- pour des raisons justiciables (ex : se faire attraper /arrêter/surprendre /prendre /gauler /coincer /condamner /enfermer /repérer/serrer /piquer, etc.)

- en bien (ex : se faire remarquer /distinguer /couronner /acclamer /draguer /aborder, etc.)

- psychologiquement affecté :

- par tromperie (ex : se faire avoir /arnaquer /piéger/rouler/plumer, etc.)

- par remontrance (ex : se faire gronder lenguirlander /disputer/houspiller /punir /engueuler /incendier /chapitrer /tirer les oreilles, etc.)

- par injure (ex: se faire huer /siffler /insulter/traiter de /injurier lagonir /persécuter /humilier /harceler, etc.)

- physiquement affecté (par violence physique) :

- sans entraîner la mort (ex : se faire agresser /frapper/gifler /poignarder/tabasser /violer lenlever /assommer /battre /renverser/fouetter/casser la gueule /bousculer létendre /piquer /lyncher lenterrer /fracasser léventrer /mordre larracher un bras /couper un doigt lattaquer /piétiner /martyriser, etc.) 
- pouvant entraîner la mort (ex: se faire tuer lassassiner labattre légorger lécraser /massacrer /dévorer /pendre/fusiller /lapider/brûler /écharper/zigouiller /électrocuter /mitrailler, etc.).

\subsection{Se faire spontané}

À la différence des valeurs de se faire que nous venons d'étudier, où le sujet représente un animé, il existe des occurrences de se faire passif dont le référent du sujet est un inanimé. On trouve ce type de construction uniquement à l'écrit, avec un nombre relativement limité de verbes, qui indiquent le surgissement d'un bruit, d'un son, d'une parole, etc. :

(76) L'influence italienne se fait sentir si fort au nord lointain de l'Europe. (D’Ormesson. J., Le bonheur à San Miniato, 1987, p. 73)

(77) Le tonnerre a commencé à se faire entendre au loin. (Djian. P., 37.2 le matin, 1985, p. 220)

(78) Le cortège (...) s'arrêta soudain : des gémissements se faisaient entendre. (D'Ormesson. J., Le vent du soir, 1985, p. 86)

(79) Les premières douleurs se firent sentir le jeudi dans la journée. (D’Ormesson. J., Le vent du soir, 1985, p. 123)

Du point de vue sémantico-syntaxique, cette construction exprime un sens passif en raison de la possibilité d'être paraphrasée par un passif canonique (ex. 80) et le complément d'agent n'est jamais exprimé : son expression rend la phrase déviante (ex. 81) :

(80) Les premières douleurs sont (res)senties le jeudi dans la journée.

(81) *Des gémissements se faisaient entendre par nous.

Sur le plan pragmatique, le sujet est presque toujours exprimé par un SN, rarement par un pronom, ce qui nous conduit à voir dans cette construction une structure rhématique, car l'expression pronominale du sujet privilégie l'interprétation à sujet animé, dans une structure topicalisante :

(82) ?Ils se faisaient entendre

De plus, les contextes dans lesquels elle apparaît présentent toujours une situation où surgit un bruit ou apparaît quelque chose, accompagnés souvent d'adverbes indiquant le surgissement: soudain, tout à coup, etc. et/ou du passé simple.

Au niveau lexical, seuls y figurent des verbes de perception ou de savoir, comme entendre (ouï), sentir (odorat, toucher, perception en général), remarquer (vue), savoir (connaissance), etc. Ainsi, lorsque se faire est construit avec un sujet inanimé et avec un verbe autre que ceux sus-mentionnés, on a alors affaire à une personnification, comme dans l'exemple suivant où la présence de l'adjectif gentil donne en plus des traits humains ou animés au référent du sujet :

(83) Notre gentil projet s'est fait adopter dans sa première mouture (Labelle, $2002: 16$ )

Comme l'indique Kokutani (2005), la construction en se faire à sujet inanimé a une lecture « spontanée », à l'instar de la diathèse spontanée du japonais qui est décrite comme une diathèse où «l'événement signifié par le verbe se réalise de lui-même ou spontanément ou à cause de la nature du sujet ». Cette lecture se caractérise également par la présence d'un verbe de perception (voir, entendre, sentir, ressentir) ou attendre, un sujet non-animé et un complément d'agent difficile.

$\mathrm{Au}$ niveau sémantico-syntaxique, cette construction est proche de la construction pronominale neutre, dans le sens où elle décrit une action qui part du sujet sans cause apparente :

(84) Il s'est levé de sa chaise

(85) Elle s'est jetée par la fenêtre

Elle partage également avec la construction passive en se faire l'aspect inaccompli et inchoatif, en raison de la possibilité de s'associer avec des semi-auxiliaires aspectuels :

(86) a. Le tonnerre a commencé à se faire entendre au loin

b. Des gémissements commençaient à se faire entendre 
c. Les premières douleurs commencèrent à se faire sentir le jeudi dans la journée

Cet aspect reste néanmoins inchoatif (marqué par le semi-auxiliaire commencer à) car cette construction s'associe plus difficilement avec d'autres semi-auxiliaires marquant par exemple le déroulement, l'achèvement du procès ou le passé proche (comme : être en train de, terminer de, venir de, etc.), en effet :

(87) a. ?Le tonnerre est en train de/vient de se faire entendre au loin

b. ?Des gémissements sont en train de/viennent de se faire entendre

c. ?Les premières douleurs sont en train de/viennent de se faire sentir

Si l'on compare cette construction à sa contrepartie sans verbe faire (donnant lieu à la construction pronominale passive), on perd alors l'aspect inchoatif :

(88) a. Le tonnerre s'est entendu au loin

b. Des gémissements s'entendaient

c. Les premières douleurs se sentirent le jeudi dans la journée

On voit ainsi que les éléments constituant cette construction se répartissent chacun différentes valeurs : le verbe à l'infinitif marque la perception (par ex. du grondement de tonnerre, dans : Le tonnerre se fit entendre) par un tiers non exprimé et l'auxiliaire se faire indique l'apparition spontanée (du grondement) combiné à l'aspect inchoatif.

\section{Conclusion}

À travers ce travail, nous avons tenté de mettre au jour le passage de se faire à lecture causative vers une lecture dérivée passive. Nous avons ainsi montré qu'à partir de la grammaticalisation de faire dans une structure causative, l'association avec la particule réflexive se, aboutit à une réanalyse où se faire devient auxiliaire de passivation.

Cette valeur passive de se faire la place d'une part en complémentarité avec le passif canonique puisque ce dernier est impossible avec les verbes doublement transitifs et, d'autre part, la met en concurrence avec le passif canonique. Ce passif concurrent remporte en outre un grand succès depuis le milieu du $20^{\mathrm{e}}$ siècle et constitue une construction très moderne. En effet, plusieurs caractéristiques le distinguant du passif canonique ont fait du passif en se faire une structure performante: l'aspect (inaccompli) de la construction, la manifestation du sens figuré du verbe à l'infinitif et la (forte) implication du référent du sujet dans le procès.

Enfin, nous avons également vu que la valeur spontanée de se faire à sujet inanimé, souvent oubliée, la place aux côtés de la construction réflexive neutre.

\section{Références}

Bat-Zeev Shyldkrot, H. (1999). Analyse sémantique d'une forme passive complémentaire : se laisser. Langages, 135, 63-74.

Bezinska, Y. (2008). Grammaticalisation et acquisition du prédicat complexe faire + Vinf. Syntaxe et sémantique des prédicats, Lidil, 37, 149-174.

Blanche-Benveniste C. (2007). Les énoncés à causatifs réfléchis. In Rousseau, A., Bottineau, D \& Roulland, D. (éds), L'énoncé réfléchi, Rennes, Presses universitaires de Rennes, 155-173.

Carlier, A. (2002). Les propriétés aspectuelles du passif. Cahiers Chronos, 10, 41-63.

Dik, S.C. (1980). Studies in Functional Grammar. London/New York : Academic Press.

François, J. (2001). Désémantisation verbale et grammaticalisation, (se) voir employé comme outil de redistribution des actants. Syntaxe \& Sémantique, 2, 159-175.

Frei, H. (2011). La grammaire des fautes. Presses Universitaires de Rennes. 
Gaatone, D. (1983). Le désagréable en syntaxe. Revue Romane, 18, 161-174.

Haspelmath, M. (1999). Why is grammaticalization irreversible ? Linguistic, 37.6, 1043-68.

Hopper, P.J. \& Traugott, E.C. (1993). Grammaticalization. Cambridge University Press.

Kokutani, S. (2005). Sur l'analyse unie de la construction 'se faire + infinitif' en français. In Bat-Zeev Shyldkrot, H. \& Le Querler, N. (éds), Les périphrases verbales, Lingvisticae Investigationes Supplementa, 25, 209-227.

Kupferman, L. (1995). La construction passive en se faire. Journal of French Language Studies, 5, 57-83.

Kurylowicz, J. (1965). The evolution of grammatical categories. Diogenes, 13 (51), 55-71. Repris in Kurylowicz, J. (1975). Esquisses linguistiques 2. Munich: W. Fink, 38-54.

Labelle, M. (2002). The French non canonical passive in se faire. In Shosuke, H., Palek, B. \& Fujimura O. (eds), Proceedings of Linguistics and Phonetics. Tokyo : Charles University Press and Meikai University, 1-26.

Lamiroy, B. (1999). Auxiliaires, langues romanes et grammaticalisation. Langages, 135, 33-45.

Lamiroy, B. et al. (2010). Les expressions verbales figées de la francophonie. Ophrys, Paris.

Langacker, R. (1977). Syntactic Reanalysis. In Li, C. (ed.), Mechanisms of Syntactic Change, Austin: University of Texas Press, $57-139$.

Le Bellec, C. (2013). La construction passive en se faire: une forme concurrente et complémentaire du passif canonique. Journal of French Language Studies (publié en ligne).

Lehmann, C. (1995). Thoughts on grammaticalization. Munich : Lincom-Europa.

Marchello-Nizia, C. (2006). Grammaticalisation et changement linguistique. Bruxelles : De Boeck.

Meillet, A. (1912). L'évolution des formes grammaticales. Linguistique historique et linguistique générale. Paris, Champion : 130-148.

Novakova, I. (2008). La construction se faire + Vinf : analyse fonctionnelle. Représentations du sens linguistique, IV, Helsinki, Finlande.

Raineri, S. (2010). Analyse contrastive français-anglais du passif dans une perspective constructionnelle: Sens et fonction de BE Ven, ETRE Vé, GET Ven et SE FAIRE Ver, Thèse de doctorat, Université Sorbonne NouvelleParis 3.

Spang-Hanssen, E. (1967). Quelques périphrases passives du français moderne. Actes du $14^{\text {ème }}$ Congrès des Romanistes Scandinaves dédiés à Holger Sten. Revue Romane, $\mathrm{n}^{\circ}$ spécial 1, Copenhague, Akademisk Forlag, 139-147.

Tasmowski, L. \& van Oevelen, H. (1987). Le causatif pronominal. Revue Romane 22, 40-58.

Traugott, E. (1980). Meaning-change in the development of grammatical markers. Language Science, 2, 44-61.

Vet, C. (1991). Les constructions causatives et réfléchies en français. In Kremer, D., Actes du XVIIIe Congrès International de Linguistique et de Philologie Romanes, Tome II, Tübigen : Max Niemeyer Verlag, 571-581.

Zribi-Hertz, A. (1982). La construction 'se-moyen' du français et son statut dans le triangle : moyen-passif-réfléchi. Linguisticae Investigationes VI-2, 345-401.

\footnotetext{
${ }^{1}$ Ce comportement est parallèle à celui du verbe avoir qui, lorsqu'il est auxiliaire, perd son contenu lexical pour se convertir, dans le groupe verbal à un temps composé, en un élément grammatical indiquant l'aspect accompli, par exemple: J'ai acheté une voiture (= aspect accompli de l'auxiliaire avoir) vs. J'ai une voiture (= je possède une voiture pour le sens plein d'avoir).

${ }^{2}$ Faire est ainsi considéré comme un auxiliaire, car il a des propriétés morphosyntaxiques analogues à celles des auxiliaires être et avoir. Néanmoins, il en diffère dans la mesure où il modifie la valence verbale, en introduisant un argument supplémentaire, c'est pourquoi on parle souvent de « semi-auxiliaire ».

${ }^{3}$ Cet exemple apparaît sur le lien qui mène à la vidéo, puis une fois sur le site où figure la vidéo, le texte décrivant la vidéo est au passif canonique, ce qui indique que la forme en se faire fonctionne comme une sorte d'appel à
} 
l'internaute, pour l'attirer; une fois sur le site, il n'a plus besoin d'appât et la forme canonique marque alors cette présentation des faits bruts.

${ }^{4} \mathrm{Si}$ le passif en être admet les deux lectures (propre et figurée), en revanche, le passif en se faire n'admet que la lecture au sens figuré. En effet, la phrase : Il a été pincé par la police peut avoir une interprétation au sens propre ou au sens figuré selon le contexte en jeu, tandis que: Il s'est fait pincer par la police ne peut recevoir que l'interprétation au sens figuré (au sens d'attraper), la lecture au sens propre est exclue (où pincer = saisir entre les doigts).

${ }^{5}$ À travers notre exploration d'occurrences dans Frantext, nous remarquons que ces exemples de verbes pris au sens figuré n'apparaissent que vers le milieu du $20^{\mathrm{e}}$ siècle, ce qui nous donne un indice pour la période de grammaticalisation de cette construction (voir section 1.2.). 